\title{
Collective Expansion in High Energy Heavy Ion Collisions
}

I. G. Bearden, ${ }^{1}$ H. Bøggild, ${ }^{1}$ J. Boissevain, ${ }^{2}$ J. Dodd,${ }^{3}$ B. Erazmus, ${ }^{4}$ S. Esumi, ${ }^{5}$ C. W. Fabjan, ${ }^{6}$ D. Ferenc, ${ }^{7}$ D. E. Fields ${ }^{2}{ }^{\text {A. Franz }},{ }^{6}$ J. J. Gaardh $ø j e,{ }^{1}$ A. G. Hansen, ${ }^{1}$ O. Hansen, ${ }^{1}$ D. Hardtke,${ }^{8}$ H. van Hecke, ${ }^{2}$ E. B. Holzer, ${ }^{9}$ T. J. Humanic,${ }^{8}$ P. Hummel, ${ }^{9}$ B. V. Jacak, ${ }^{2}$ R. Jayanti, ${ }^{8}$ H. Kalechofsky, ${ }^{8}$ M. Kaneta, ${ }^{5}$ M. Kopytine, ${ }^{2}$ Y. Y. Lee, ${ }^{8}$ M. Leltchouk, ${ }^{3}$ T. Ljubicic, ${ }^{7}$ B. Lörstad,${ }^{10}$ N. Maeda, ${ }^{5}$ R. Malina, ${ }^{9}$ A. Medvedev, ${ }^{3}$ M. Murray, ${ }^{11}$ S. Nishimura, ${ }^{5}$ H. Ohnishi,${ }^{5}$ G. Paic, ${ }^{4}$ S. U. Pandey,${ }^{8}$ F. Piuz,${ }^{6}$ J. Pluta, ${ }^{4}$ V. Polychronakos,${ }^{12}$ M. Potekhin, ${ }^{3}$ G. Poulard,${ }^{6}$ D. Reichhold ${ }^{8}$ A. Sakaguchi, ${ }^{5}$ J. Simon-Gillo, ${ }^{2}$ J. Schmidt-Sørensen, ${ }^{10}$ W. Sondheim, ${ }^{2}$ M. Spegel, ${ }^{6}$ T. Sugitate,${ }^{5}$ J. P. Sullivan, ${ }^{2}$ Y. Sumi,${ }^{5}$ W. J. Willis, ${ }^{3}$ K. Wolf,${ }^{11}$ N. Xu, ${ }^{2}$ and D. S. Zachary ${ }^{8}$

(The NA44 Collaboration)

${ }^{1}$ Niels Bohr Institute, DK-2100 Copenhagen, Denmark

${ }^{2}$ Los Alamos National Laboratory, Los Alamos, New Mexico 87545

${ }^{3}$ Department of Physics, Columbia University, New York, New York 10027

${ }^{4}$ Nuclear Physics Laboratory of Nantes, 44072 Nantes, France

${ }^{5}$ Hiroshima University, Higashi-Hiroshima 739, Japan

${ }^{6}$ CERN, CH-1211 Geneva 23, Switzerland

${ }^{7}$ Rudjer Boskovic Institute, Zagreb, Croatia

${ }^{8}$ Department of Physics, The Ohio State University, Columbus, Ohio 43210

${ }^{9}$ Technical University, A-1040 Vienna, Austria

${ }^{10}$ Department of Physics, University of Lund, S-22362 Lund, Sweden

${ }^{11}$ Texas A\&M University, College Station, Texas 77843

${ }^{12}$ Brookhaven National Laboratory, Upton, New York 11973

(Received 4 November 1996)

\begin{abstract}
Transverse mass spectra of pions, kaons, and protons from the symmetric heavy-ion collisions $200 A \mathrm{GeV} \mathrm{S}+\mathrm{S}$ and $158 \mathrm{~A} \mathrm{GeV} \mathrm{Pb}+\mathrm{Pb}$, measured in the NA44 focusing spectrometer at CERN, are presented. The mass dependence of the slope parameters provides evidence of collective transverse flow from expansion of the system in heavy-ion induced central collisions. [S0031-9007(97)02697-5]
\end{abstract}

PACS numbers: 25.75.Ld

The purpose of studying ultrarelativistic heavy-ion collisions is to understand the nature of hadronic matter under extreme conditions. Specifically, we are interested in a new form of matter, quark-gluon plasma, which may be produced in such collisions. Transverse momentum distributions are one of the most common tools used in studying high energy collisions. This is because the transverse motion is generated during the collision and hence is sensitive to the dynamics. More than 45 years ago, Fermi proposed a statistical method [1] to understand the results of high energy hadron-hadron collisions. Because of saturation of the phase space, the multiparticle production resulting from the high energy elementary collisions is consistent with a thermal description [1-3]. In heavy-ion collisions hydrodynamical behavior, that is, local thermal equilibrium and collective motion, may be expected because of the large number of secondary scatterings.

It is now possible to identify and quantitatively measure the collective motion by systematic studies of results from different collision systems, using light ( $\mathrm{Si}$ at BNL and $\mathrm{S}$ at CERN) and heavy ( $\mathrm{Au}$ at $\mathrm{BNL}$ and $\mathrm{Pb}$ at CERN) ion beams [4-6]. A high degree of nuclear stopping and a strong Coulomb effect (also due to the high stopping) have already been reported in $\mathrm{Pb}+\mathrm{Pb}$ central collisions $[7,8]$. In this Letter, we present transverse momentum distributions of pions, kaons, and protons, measured in the
NA44 spectrometer, from $\mathrm{Pb}+\mathrm{Pb}$ and $\mathrm{S}+\mathrm{S}$ collisions. Results of calculations from a hydrodynamical model [5] will be used to aid in this analysis.

The NA44 magnetic focusing spectrometer consists of two room-temperature dipoles and three superconducting quadruples. Particles originating from the target are focused at a plane about ten meters downstream and detected by a tracking system consisting of a pad chamberstrip chamber-scintillator hodoscope complex. Particle identification is done with two threshold Cherenkov counters and two highly segmented TOF hodoscopes. The phase-space coverage (transverse momentum $p_{T}$ vs rapidity $y$ ) is determined by the combination of the spectrometer angle (relative to the beam direction) and the nominal momentum setting of the magnets. The momentum resolution is typically $\sigma_{p} / p \leq 0.2 \%$ and the TOF counters have an average time resolution of $100 \mathrm{ps}$. More details of the spectrometer can be found elsewhere [9].

The spectrometer momentum range is $\pm 20 \%$ around the nominal values of 4 and $8 \mathrm{GeV} / c$. For kaons and protons, the $8 \mathrm{GeV} / c$ setting was used and the rapidity coverage is (2.5-3.4) and (2.4-2.8) for kaons and protons, respectively. Two angular settings (44 and $130 \mathrm{mrad}$ ) were utilized in order to cover the transverse momentum range $0 \leq p_{T} \leq 1.6 \mathrm{GeV} / c$. For the pion data, the 4 and $8 \mathrm{GeV} / c$ low angle data were combined (for details of 
this procedure, see Ref. [7]) and the overall rapidity window is $(3.0-4.1)$. For a symmetric colliding system, all particles are near midrapidity $y_{\text {c.m. }}=2.9$ and $y_{\text {c.m. }}=3.0$ for the beam energies of 158 and $200 \mathrm{~A} \mathrm{GeV}$. The $10 \%$ and $6.4 \%$ most central collisions were selected from the $\mathrm{S}+\mathrm{S}$ and $\mathrm{Pb}+\mathrm{Pb}$ data, respectively.

For the $158 \mathrm{AGeV} \mathrm{Pb}+\mathrm{Pb}$ central collisions, the midrapidity transverse mass distributions, $\left(1 / m_{t}\right) d N / d m_{T}$ as a function of $m_{T}-m\left(m_{T}=\sqrt{p_{T}^{2}+m^{2}}\right)$, for both signs of pions, kaons, and protons are shown in Fig. 1 as open circles. The dashed lines in Fig. 1 are fits to the function

$$
\frac{1}{m_{T}} \frac{d N}{d m_{T}}=A \exp \left(-\frac{m_{T}}{T}\right),
$$

with $A$ an arbitrary constant and $T$ characterizing the slope of the distribution. For kaons and protons, the fitting is done in the range $0 \leq m_{T}-m \leq 1 \mathrm{GeV}$. In order to exclude the $m_{T}$ range populated heavily by resonance decays, the fitting range for pions is $m_{T}-m \geq 0.25 \mathrm{GeV}$.

The systematic errors for kaon and proton spectra are estimated to be less than 5\%, for pions less than $15 \%$. The larger uncertainties in the pion spectra mainly arise from two sources. One of these is the acceptance correction which was done neglecting the rapidity dependence of the slope parameter. However, the measured [10] pion mean $p_{T}$ from $\mathrm{Pb}+\mathrm{Pb}$ collisions does not vary significantly within $3.0 \leq y \leq 4.5$, validating this assumption. We estimate an error of $10 \%$ in the final pion slope parameter from this source. The other contribution is the fitting range. Because of resonance decays, a pion spectrum over a wide transverse momentum range is normally not exponential with respect to the transverse mass $m_{T}$. It can be seen in Fig. 1 that the exponential fits represent the data very well for all cases except for pions in the low $p_{T} \leq 0.2 \mathrm{GeV}$ region. The discrepancies between data and the fits in this region are most likely due to the resonance decays [11-15].

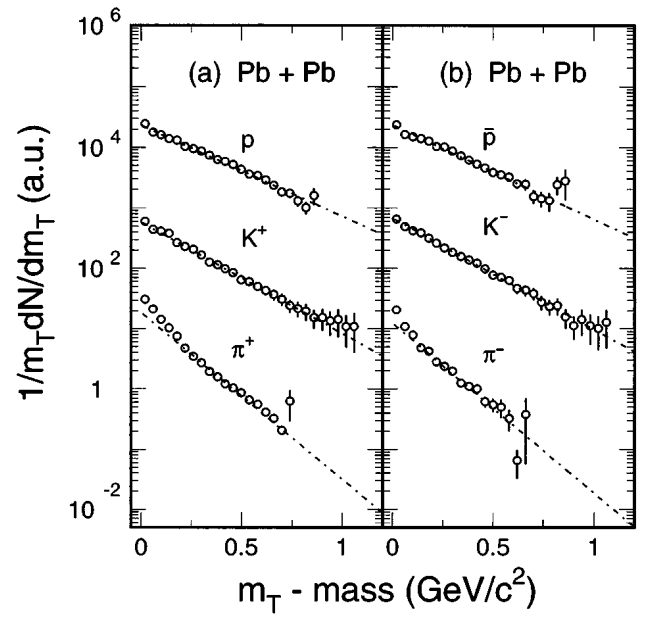

FIG. 1. Transverse mass distributions for pions, kaons, and protons from $158 \mathrm{AGeV} \mathrm{Pb}+\mathrm{Pb}$ central collisions. Dashed lines represent the exponential fits.
The slope parameter $T$, from the $\mathrm{Pb}+\mathrm{Pb}$ collisions at $\sqrt{s}=17.2 A \mathrm{GeV}$ increases with particle mass and the trend is independent of the sign of the particle under consideration. Slope parameters of these particles were also determined from $\sqrt{s}=19.4 A \mathrm{GeV} / c \mathrm{~S}+\mathrm{S}$ central collisions measured in the NA44 spectrometer. The behavior is similar to that described above for $\mathrm{Pb}+\mathrm{Pb}$. The values of slope parameter from $p+p, \mathrm{~S}+\mathrm{S}$, and $\mathrm{Pb}+\mathrm{Pb}$ collisions are listed in Table I. The $p+p$ $(\sqrt{s}=23 \mathrm{GeV})$ results are taken from Refs. [16,17].

In order to visualize the systematic trends in the data, we summarize the slope parameters for pions, kaons, and protons from the three collision systems in Fig. 2. On the left are the slopes for positive particles and on the right for negative particles. Both particles and antiparticles exhibit similar behavior. A distinct difference between the elementary $p+p$ and heavy-ion collisions is reflected in the slope parameters. While for $p+p$ the slope parameters (triangles) remain constant as a function of the particle mass, these parameters increase with increasing mass for heavy-ion collisions. Furthermore, for a given mass, the heavier the colliding system, the higher the slope parameter. It is particularly interesting to observe that all curves converge to a point about $T \approx 140 \pm 15 \mathrm{MeV}$ as the particle mass approaches zero [18]; this range is indicated by the shaded bars in the Fig. 2. It is worth noting that a plot similar to Fig. 2 can be made for data at AGS energies $\left(E_{\text {beam }} \approx 10-15 A\right)$ [19]. Remarkably, the characteristic slope is also about $145 \mathrm{MeV}$. On the other hand, at lower beam energies of $E_{\text {beam }} \approx 0.2-5 A \mathrm{GeV}$, one finds that this $T$ decreases dramatically as the beam energy decreases [20-23].

In a hydrodynamical picture, matter flows, i.e., particles of different mass all move with the same velocity. Classically, the collective kinetic energy will then depend on the particle mass: Particles with higher mass will carry more energy. The experimental slope parameter measures the particle energy which contains both thermal (random) and collective (due to secondary collisions among the produced particles, or rescattering) contributions. The intrinsic freeze-out temperature, namely, the temperature when the particles cease to interact with each other, is determined by the thermal motion. As rescattering is not important in $p+p$ collisions, the slope parameter should be due only to the thermal motion and thus may reflect the true freeze-out temperature. This interpretation is strengthened by the observation that the slope parameter remains nearly constant (around $140-150 \mathrm{MeV}$ ) for all particles from the $p+p$ data. When the size of the colliding nuclei increases, so does the number of rescatterings and, as a consequence, collective motion develops. The rise of $T$ with particle mass seen in Fig. 2 is indeed characteristic of such hydrodynamical behavior. The cascade code RQMD [24] predicts a similar trend in the slope parameters as a function of particle mass. However, it systematically underestimates the values for kaons and protons for all collision systems. 
TABLE I. Slope parameters $T$ (in MeV unit) of pions, kaons, and protons for $p+p, \mathrm{~S}+\mathrm{S}$, and $\mathrm{Pb}+\mathrm{Pb}$ colliding systems at CERN energies. Error bars are statistical only. The systematic errors for kaons and protons are less than 5\% while for pions they are less than $15 \%$.

\begin{tabular}{|c|c|c|c|c|c|c|}
\hline & $\pi^{+}$ & $K^{+}$ & $p$ & $\pi^{-}$ & $K^{-}$ & $\bar{p}$ \\
\hline$p$ & $139 \pm 13$ & $139 \pm 15$ & $148 \pm 20$ & $141 \pm 8$ & $150 \pm 10$ & $151 \pm 16$ \\
\hline$S$ & $148 \pm 4$ & $180 \pm 8$ & $208 \pm 8$ & $148 \pm 4$ & $180 \pm 7$ & $190 \pm 7$ \\
\hline $\mathrm{Pb}$ & $156 \pm 6$ & $234 \pm 6$ & $238 \pm 7$ & $154 \pm 8$ & $235 \pm 7$ & $278 \pm 9$ \\
\hline
\end{tabular}

The correlation between the slope parameter and particle mass $m$ may be described qualitatively by the relationship

$$
T=T_{\mathrm{fo}}+m\left\langle v_{T}\right\rangle^{2} .
$$

The parameters $T_{\text {fo }}$ and $\left\langle v_{T}\right\rangle$ can be identified with the freeze-out temperature and the averaged collective flow velocity, respectively. Fitting the $p+p$ data with this expression, one finds a temperature of around $140 \mathrm{MeV}$ and a flow velocity consistent with zero for both particles and antiparticles. On the other hand, for $\mathrm{S}+\mathrm{S}$ and for $\mathrm{Pb}+\mathrm{Pb}$, the fits give nearly the same temperature but a large velocity. Results of the fits are presented in Table II, where the quoted errors are statistical only. Because of the assumption of a linear mass dependence used in the fit, the uncertainty in the resulting velocity is estimated to be $15 \%$. The difference between the proton and antiproton freeze-out temperatures from the $\mathrm{Pb}+\mathrm{Pb}$ collisions is less than $1.5 \sigma$. In $p+p$ collisions, the density of produced particles is low, insufficient to produce an appreciable collective flow. Therefore, the slope parameters show no dependence on the particle mass $[25,26]$. Conversely, for heavy colliding systems the density of produced particles is rather high, giving rise to significant rescattering, and thus collective transverse motion. In Ref. [27], the authors discuss a similar relationship between slope parameter and particle mass within the framework of a hydrodynamical model. There the $\left\langle\boldsymbol{v}_{T}\right\rangle$ is interpreted as the mean expansion velocity at the freezeout surface.

Using the hydrodynamical model of Refs. [4,5], the transverse momentum distributions are calculated with the form

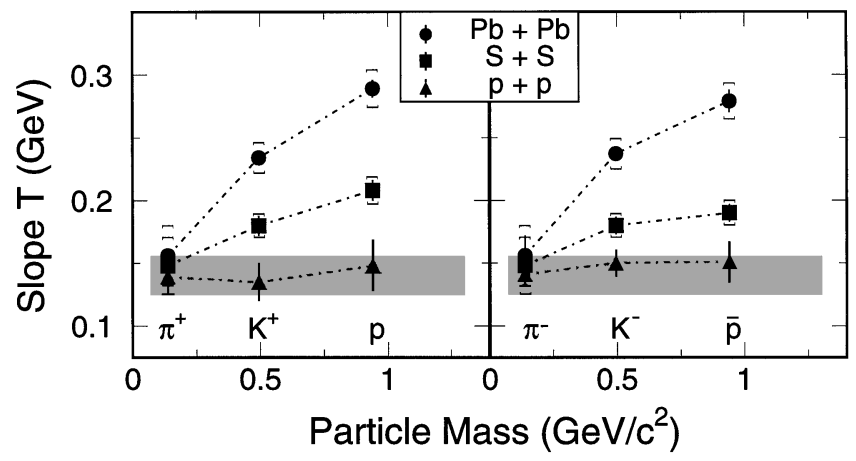

FIG. 2. Slope parameter $T$ as a function of particle mass. The $p+p$ results are taken from Refs. [16,17]. The systematic errors are shown by the vertical brackets. The shaded band shows the range where the slopes converge at zero mass.

$$
\begin{aligned}
\frac{d N}{m_{T} d m_{T}} \propto & \int_{0}^{R} r \operatorname{drm}_{T} I_{0}\left[\frac{p_{T} \sinh (\rho)}{T_{0}}\right] \\
& \times K_{1}\left[\frac{m_{T} \cosh (\rho)}{T_{0}}\right],
\end{aligned}
$$

where $\rho=\tanh ^{-1} \beta_{r}, \beta_{r}(r)=\beta(r / R)^{\alpha}$ with $\alpha=1$, and $I_{0}, K_{1}$ are modified Bessel functions. Two free parameters, the temperature $T_{0}$ and the surface transverse velocity $\beta_{s}$, determine the shape of the $m_{T}$ spectra. The calculated result is used to fit all measured pions, kaons, and protons, from a given reaction.

Figure 3 shows the results of the fitting as solid lines which agree well with experimental data. For kaons and protons, within statistical errors, no dependence on the fitting range was found. However, as mentioned earlier, pion distributions are affected by resonance decays at low $p_{T}$. In order to account for this effect, resonance decays are included in the calculations. In principle there are two ways to estimate the resonance decays. Assuming a thermal population for the heavy resonances, the pion distribution can be calculated [5,6]. This implicitly assumes a chemical equilibrium and the unmeasured longitudinal distributions of these resonances have to be assumed as well. An alternative way to evaluate the resonance effect is to use a cascade code like RQMD [28]. We calculate the ratio of the pions with and without resonance decays as a function of transverse mass. This ratio is then applied to the thermal model result. Thermal equilibrium is implicitly assumed in this approach, but this is much less strict than the chemical equilibrium assumption, although all assumptions that went into the cascade model calculations are in principle included in the ratio estimation. In the RQMD model [28], we find that at least $50 \%$ of final midrapidity pions are from resonance decays for both $\mathrm{Pb}+\mathrm{Pb}$ and $\mathrm{S}+\mathrm{S}$ collisions.

With the intrinsic temperature fixed at $T_{0}=140 \mathrm{MeV}$, the maximum velocities are found to be 0.6 and $0.41 \mathrm{c}$ for $\mathrm{Pb}+\mathrm{Pb}$ and $\mathrm{S}+\mathrm{S}$ collisions, respectively. (These

TABLE II. Fitted [Eq. (2)] temperature parameter $T_{\text {fo }}$ (in $\mathrm{MeV}$ unit) and averaged velocity parameter $\left\langle\boldsymbol{v}_{T}\right\rangle$ for $p+p$, $\mathrm{S}+\mathrm{S}$, and $\mathrm{Pb}+\mathrm{Pb}$ colliding systems at the CERN energies. Error bars are statistical only. The "+" and "-" signs are for positive and negative particles, respectively.

\begin{tabular}{clccc}
\hline \hline & \multicolumn{1}{c}{$T_{\mathrm{fo}}^{+}$} & $\left\langle v_{T}\right\rangle^{+}(c)$ & \multicolumn{1}{c}{$T_{\mathrm{fo}}^{-}$} & $\left\langle v_{T}\right\rangle^{-}(c)$ \\
\hline$p+p$ & $136 \pm 15$ & $0.09 \pm 0.17$ & $140 \pm 10$ & $0.12 \pm 0.15$ \\
$\mathrm{~S}+\mathrm{S}$ & $138 \pm 5$ & $0.28 \pm 0.10$ & $142 \pm 5$ & $0.24 \pm 0.10$ \\
$\mathrm{~Pb}+\mathrm{Pb}$ & $140 \pm 7$ & $0.41 \pm 0.11$ & $167 \pm 13$ & $0.36 \pm 0.14$ \\
\hline \hline
\end{tabular}




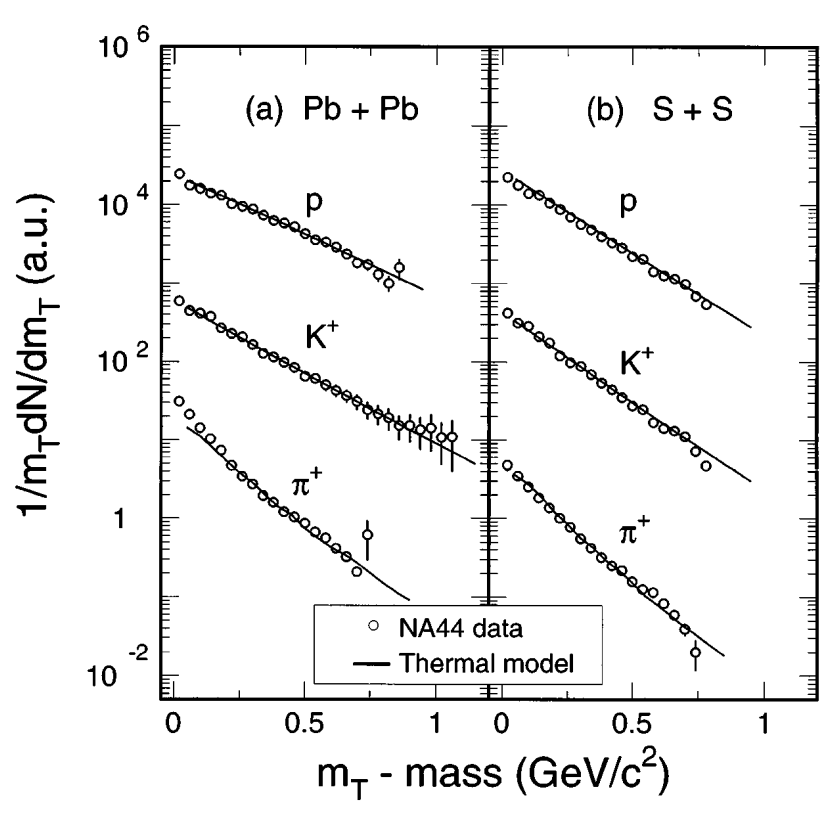

FIG. 3. Transverse mass distributions for pions, kaons, and protons (open circles) compared with results of a thermal model calculation (lines). Resonance decays are included in the pion spectra, and resonance effects on kaon and proton spectra are neglected.

values correspond to average transverse flow velocities of 0.4 and $0.27 c$.) The fit results show an anticorrelation between the temperature $T_{0}$ and the maximum velocity $\beta_{s}$. Within a temperature range $100 \leq T_{0} \leq 150 \mathrm{MeV}$, the fits are equally good. The choice of $T_{0}=140 \mathrm{MeV}$ is based on Fig. 2 and Refs. [5,24,29,30]. The success of fitting the different mass particle spectra with one expanding source lends credence to the naive interpretation of the slope trend as evidence of collective expansion.

Note that Eq. (2) does not include any details of the freeze-out process. However, the hydrodynamical model Eq. (3) includes a specific shape of the freezeout surface Ref. [5]. The extracted values of the mean velocity are similar for the two models. From the data we cannot tell whether this is accidental or the single-particle distributions are not sensitive to the freeze-out surface.

In summary, we report NA44 results on transverse mass spectra of pions, kaons, and protons from the symmetric system of $200 A \mathrm{GeV} \mathrm{S}+\mathrm{S}$ and $158 \mathrm{AGeV} \mathrm{Pb}+\mathrm{Pb}$ collisions. While no collective effect is found in $p+p$ reactions, collective transverse flow is clearly evident in the heavy-ion collisions. The strongest transverse flow is found in the $\mathrm{Pb}+\mathrm{Pb}$ system. The reason for this trend is that a high nuclear stopping is reached in heavy-ion collisions and particles experience many collisions before they leave the system.

We are grateful to the staff of the CERN PS-SPS accelerator complex for their excellent work. We thank the technical staff at CERN and the collaborating institutes for their valuable contribution. We are also grateful for the support given by the Austrian Fonds zur Förderung der Wissenschaftlichen Forschung; the Science Research Council of Denmark; the Japanese Society for the Promotion of Science; the Ministry of Education, Science and Culture, Japan; the Science Research Council of Sweden; the National Science Foundation, the U.S. Department of Energy, and the W. B. Keck Foundation.

[1] E. Fermi, Prog. Theor. Phys. 5, 570 (1950).

[2] E. L. Feinberg, Sov. Phys. Usp. 14, 455 (1972).

[3] E. V. Shuryak, Sov. J. Nucl. Phys. 20, 295 (1975).

[4] S. K. Lee, U. Heinz, and E. Schnedermann, Z. Phys. C 48, 625 (1990).

[5] E. Schnedermann, J. Sollfrank, and U. Heinz, Phys. Rev. C 48, 2462 (1993).

[6] P. Braun-Munzinger, J. Stachel, J. Wessels, and N. Xu, Phys. Lett. B 344, 43 (1995); ibid. 356, 1 (1996).

[7] NA44 Collaboration, H. Bøggild et al., Phys. Lett. B 372, 339 (1996).

[8] NA44 Collaboration, I. G. Bearden et al., Phys. Lett. B 388, 431 (1996).

[9] H. Beker et al., Phys. Lett. B 302, 510 (1993).

[10] NA49 Collaboration, P. G. Jones et al., in Proceedings of the Conference on Quark Matter, Heidelberg, Germany, 1996.

[11] NA44 Collaboration, H. Bøggild et al., Z. Phys. C 69, 621 (1996).

[12] E814 Collaboration, J. Barrette et al., Phys. Lett. B 351, 93 (1995).

[13] E802 Collaboration, M. Gonin et al., Nucl. Phys. A566, 601c (1993).

[14] S. Ahmad et al., Phys. Lett. B 281, 29 (1992).

[15] T. Akesson et al., Z. Phys. C 46, 361 (1990).

[16] B. Alper et al., Nucl. Phys. B100, 237 (1975).

[17] K. Guettler et al., Nucl. Phys. B116, 77 (1976).

[18] NA44 Collaboration, N. Xu et al., in Ref. [10].

[19] E802 Collaboration, Z. Chen et al., in Proceedings of the BNL AGS Users Meeting, 1995; E802 Collaboration, T. Abbott et al., Phys. Rev. C 50, 1024 (1994); Z. Chen, Int. J. Mod. Phys. E, 2, 285 (1993).

[20] S. Nagamiya et al., Phys. Rev. C 24, 971 (1981).

[21] G. Poggi et al., Nucl. Phys. A586, 755 (1995).

[22] M. A. Lisa et al., Phys. Rev. Lett. 75, 2662 (1995).

[23] A. Jipa, J. Phys. G 22, 231 (1996).

[24] H. Sorge, Phys. Lett. B 373, 16 (1996).

[25] R. Hagedorn and J. Rafelski, Phys. Lett. 97B, 136 (1980).

[26] E. V. Shuryak and O. V. Zhirov, Phys. Lett. 89B, 253 (1980).

[27] T. Csörgó and B. Lörstad, Phys. Rev. C 54, 1390 (1996).

[28] H. Sorge, Phys. Rev. C 52, 3291 (1995).

[29] I. Ya. Pomeranchuk, Dokl. Akad. Sci. SSSR 78, 884 (1951); L. D. Landau, Izv. Akad. Nauk SSSR Ser. Fiz. 17, 51 (1953).

[30] B. Schlei et al., Phys. Lett. B 376, 212 (1996). 